\title{
Vector Overhauser magnetometer POS-4: experience and prospects of application
}

\author{
Sergey Y. Khomutov ${ }^{1, *}$, Vladimir A. Sapunov ${ }^{2, * *}$, Alexey Y. Denisov ${ }^{2,}$, Pavel B. Borodin ${ }^{3,}$, \\ Dmitry V. Kudin ${ }^{4,5}$, Roman V. Sidorov ${ }^{4}$, Alexey S. Bebnev ${ }^{3}$, Tatyana A. Cherepanova ${ }^{3,}$, An- \\ drey L. Kotikov ${ }^{4,6}$, Konstantin K. Kanonidi ${ }^{7}$, Yury G. Turbin ${ }^{8,}$, and Pavel E. Baryshev ${ }^{8}$, \\ ${ }^{1}$ Institute of Cosmophysical Research and Radio Wave Propagation FEB RAS, Paratunka, Kamchatka, \\ Russia \\ ${ }^{2}$ Ural Federal University, Ekaterinburg, Russia \\ ${ }^{3}$ Institute of Geophysics UB RAS, Ekaterinburg, Russia \\ ${ }^{4}$ Geophysical Center RAS, Moscow, Russia \\ ${ }^{5}$ Gorno-Altaisk State University, Gorno-Altaysk, Altai Republic, Russia \\ ${ }^{6}$ St.-Petersburg branch, Pushkov Institute of Terrestrial Magnetism, Ionosphere and Radio Wave Prop- \\ agation of RAS, Saint-Petersburg, Russia \\ ${ }^{7}$ Pushkov Institute of Terrestrial Magnetism, Ionosphere and Radio Wave Propagation RAS, Moscow, \\ Russia \\ ${ }^{8}$ The Arctic and Antarctic Research Institute (AARI) of the Russian Federal Service for Hydrometeo- \\ rology and Environmental Monitoring, Roshydromet, Saint-Petersburg, Russia
}

\begin{abstract}
The results of practical use of a POS-4 vector magnetometer, developed by the Research Laboratory of Quantum Magnetometry, UrFU (Yekaterinburg) and based on POS Overhauser sensors, are presented. Continuous measurements by POS-4 have been carried out at the Paratunka observatory (IKIR FEB RAS, Kamchatka) since 2015, were done at the Saint Petersburg observatory (GC RAS / IZMIRAN SPb Branch, Leningrad Region) in 20172018 and have been performed at the Arti observatory (Institute of Geophysics, UB RAS, Sverdlovsk Region) since 2020. On the new high-latitude observatory White Sea (IAGA code WSE, GC RAS / MSU, Nikolai Pertsov White Sea Biological Station, Karelia), POS-4 is used as a main variometer for magnetic measurements. In April 2019, the magnetometer was successfully used for field measurements on ice during the TRANSARCTIC expedition in the Barents Sea (AARI, Roshydromet). At the beginning of 2021 IZMIRAN started testing two POS-4 magnetometers at the Moskow observatory. According to the results of field and observatory measurements it was possible to identify the advantages and disadvantages of the magnetometer and provide the information for its developers for further modernization in order to improve its efficiency and reliability. Many years of experience in POS-4 application determine the areas where its scientific and applied usage will provide important results, for example, for magnetic measurements in the Arctic regions or for monitoring of active zones around volcanoes.
\end{abstract}

\footnotetext{
*e-mail: khomutov@ikir.ru

**e-mail: vasapunov@gmail.com
} 


\section{Introduction}

Ground-based magnetic measurements at observatories, Repeat stations and during surveys of various ranks are an important part of the study of the Earth's magnetic field. The need to obtain results with ever higher accuracy and reliability leads to new requirements for measurement technology, processing and analysis. Technical tools such as magnetometers, data collection and transmission systems are very important.

In recent decades, the development of magnetometers has made significant progress, especially with regard to devices for measuring variations and the total field intensity. However, with respect to magnetometers for measuring the full vector of magnetic induction (absolute measurements), progress is not so significant. Almost everywhere, a set of two magnetometers is used for this kind of measurement - a scalar, usually based on an Overhauser or quantum sensor, and a DI-magnetometer (DIflux) using a fluxgate sensor mounted on a nonmagnetic theodolite tube. Such a set is the standard for the observatories of the INTERMAGNET network [1]. However, with the relative stability of the results, DIflux requires manual work of a highly qualified magnetologist, has low productivity and some limitations due to the observation technology.

One of the approaches for creating an automatic magnetometer for absolute observations is the automation of DIflux. AutoDIF and GyroDIF magnetometers have been developed and are already being used in observational practice, completely new devices with an automatic laser binding system to a remote reference point, a sensor rotation system and high-precision positioning $[2,3]$. In addition, there are DIflux based on standard non-magnetic theodolites, in which manual manipulations and visual reading of the scale readings are performed automatically [4]. Together with a scalar magnetometer, an automatic or semi-automatic system for measuring the full field vector is obtained.

Another approach is based on the use of scalar sensors that measure the total field intensity $\mathrm{F}$, placed in a system of coils that create compensating or additional fields in specified directions. Such systems were developed quite a long time ago and were used at observatories for absolute measurements of the horizontal and vertical components of the vector F, but they required careful tuning, calibration and a significant amount of manual procedures and time [5].

With the advent of sensors using the Overhauser effect and new technological approaches, it became possible to significantly automate measurements and calculations, minimize methodological errors and perform measurements with a frequency comparable to ordinary variometers. In practice, this approach was implemented on the basis of the developments of L. Hegimegy (Hungary) at GEM Systems (Canada) as the dIdD GSM-19FD magnetometer [6] and in the Laboratory of Quantum Magnetometry of UrFU (QMLab, Yekaterinburg, Russia) in POS-3 and POS-4 magnetometers [7].

\section{Description of the magnetometer POS-4}

The POS-4 absolute vector magnetometer was developed in the Laboratory of Quantum Magnetometry of the Ural Federal University on the basis of a high-precision scalar Overhauser magnetometer POS-1 (registered in the state Register of the SI of the Russian Federation, No. 79451-20). The device combines absolute measurements of the total field intensity with the accuracy of a scalar magnetometer $[8,9]$ and measurements of the vertical and horizontal intensity of Earth's magnetic field [10].

The magnetometer consists of three key components: a magnetizing system, a primary probe (an Overhauser sensor) placed in it, and an electronics unit separate from the magnetic 
system. The electronics unit is connected to a computer that controls the magnetometer, processes and stores data (see figure 1).

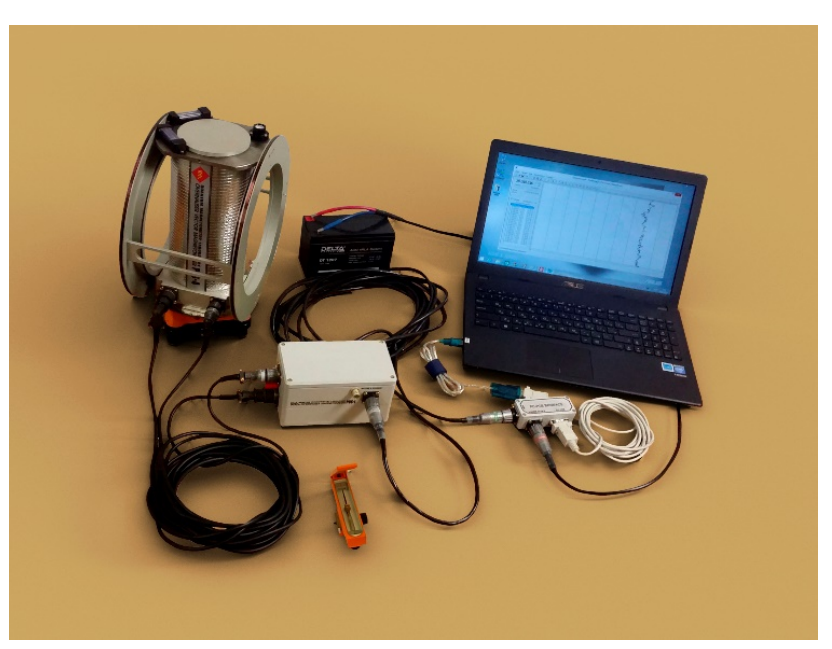

Figure 1. Assembly of the POS-4 magnetometer (from left to right: a solenoid with a coil system and a sensor, an electronics unit with a battery, an interface switching unit and a recording laptop).

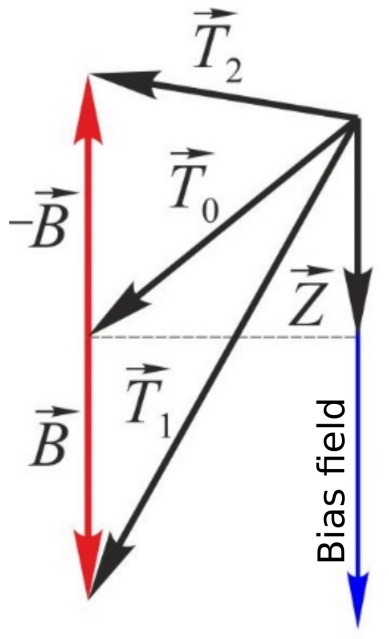

Figure 2. Field switching scheme in a vertical magnetizing system.

The magnetic field is measured by a primary probe that generates a signal of free precession of the nuclear magnetization of the working substance, followed by the calculation of the total field intensity in the electronics unit.

The magnetic system creates a magnetizing field (uniform in the sensor area) in the direction of the measured field components. The vertical magnetizing field is formed by a vertical Garrett solenoid. The verticality of the solenoid installation is ensured with high accuracy by two mutually perpendicular liquid levels at the top of the solenoid. Additionally, POS-4 contains a magnetic coil system that forms a horizontal magnetizing field. The recommended orientation (according to the criterion of the maximum sensitivity of the sensor) of this magnetization system corresponds to the choice of the direction of the axis of the coils perpendicular to the magnetic meridian plane, for example, using the reference compass. The concept of operation of POS-4 is based on measurements of the total field intensity by a scalar magnetometer placed in a magnetizing system that creates switched highly homogeneous magnetic fields. The magnetic system includes a vertical Garrett solenoid and a system of four coils that creates a horizontal field.

In each of the magnetic subsystems, a connected controlled source of stable switched current creates a magnetizing field by setting the current: $i=+I,-I, 0$. The upgraded software for POS magnetometers provides current switching and recording of the obtained scalar values of the total field intensity with subsequent calculation and visualization of the vertical $\mathrm{Z}$ and horizontal $\mathrm{H}$ components. In particular, the measurement of the vertical component (see figure 2) is carried out according to the following formulas:

$$
Z=\frac{T_{1}^{2}-T_{2}^{2}}{4 B} ; \quad B=\sqrt{\frac{T_{1}^{2}+T_{2}^{2}}{2}-T_{0}^{2}} .
$$


Similarly, the horizontal component $\mathrm{H}$ is measured. It should be noted that the "raw" data $T_{0}, T_{1}, T_{2}, T_{1}^{\prime}, T_{2}^{\prime}$ ( $T^{\prime}$ are fields measured by secondary system) are stored in the data file for the possibility of further more detailed processing.

The high absoluteness of the POS-4 vector magnetometer results are due, firstly, to the absoluteness of the POS-1 Overhauser sensor as a probe and, secondly, to the technology of the magnetic system produce based on a titanium frame.

\section{Geophysical Observatory Paratunka, IKIR FEB RAS, Kamchatka}

The POS-4 magnetometer was purchased by IKIR FEB RAS as part of the implementation of the RSF project, including to assess the possibility to upgrade the observatory's magnetic monitoring to Russian-made equipment. The tasks of testing of magnetometer with the prospect of installation at the observatory Cape Schmidt (CPS) at Chukotka were also solved.

To install the magnetometer, a small $2 \times 2 \mathrm{~m}$ wooden pavilion was built with an inner layer of expanded polystyrene for passive thermal stabilization. Such installation of the POS-4 provided full-fledged maintenance and carrying out various works, without interfering with other magnetometers of the observatory used for regular monitoring of the magnetic field. The noise influence during the generation of additional fields in the coil system and solenoid of POS-4 was also excluded. Inside the pavilion, a pillar was installed, in the lower part cast from a special low-magnetic mixture, and in the upper part made of glass blocks. There is no $220 \mathrm{~V}$ power supply to the pavilion, there is no lighting and heating. To control the thermal conditions, three digital temperature sensors DS18B20 with a sensitivity of $0.1^{\circ} \mathrm{C}$ are installed: (a) in a thermally insulated air gap around the sunken part of the pillar (to control freezing, the gap is filled with water in the spring), (b) at the height of the sensor and (c) on the housing of the electronics unit (to control overheating).

The POS-4 solenoid with a standard non-magnetic stand from theodolite is levelled using two high-precision bubble levels on its upper part. The horizontal axis of the magnetometer is oriented to the geographical East, so the values of the horizontal component are close to the current value of the component $\mathrm{Y}$ of the magnetic field. A recording laptop with a standard software POS Manager and a power supply system are installed in the technical pavilion at a distance of about $20 \mathrm{~m}$. The magnetometer timer is synchronized when the measurements are started or manually according to the laptop's system time, which is tied to UTC every hour by the institute's time server. The measurement of single total field intensity value is 2 seconds, so the full cycle (measurement of the full magnetic field vector) takes 10 seconds. The photo and diagram of the pavilion, the photo of the sensor on the pillar are shown in figure 3 , a detailed description is given in [7, 11].

Regular measurements using POS-4 were started in the spring of 2015 and continue continuously to the present. There was a short break in November 2016, when the magnetometer was unmounted for the period of work on strengthening the lower part of the pillar (strengthening was required due to the low stability of the pillar, especially during the freezing and defrosting of the surrounding soil). No special work was carried out with the magnetometer. But occasionally, with a noticeable departure of the levels, a corrective leveling of solenoid was performed. Also every year in the autumn the air gap between the ground and the pillar was insulated (in early summer, the insulation was removed).

The magnetometer POS-4 was mainly considered as a variometer and complete processing was performed for it, as for the other magnetometers of the observatory: check of raw records, cleaning from noise, calculation of baseline values and minute values. In some cases, POS-4 data was used to fill the gaps in the records of the main magnetometers, if the data of the backup devices were also missing. In figure 4 the comparison of the raw POS- 4 data with the records of the FGE-DTU fluxgate variometer, which is the main one at the observatory, 


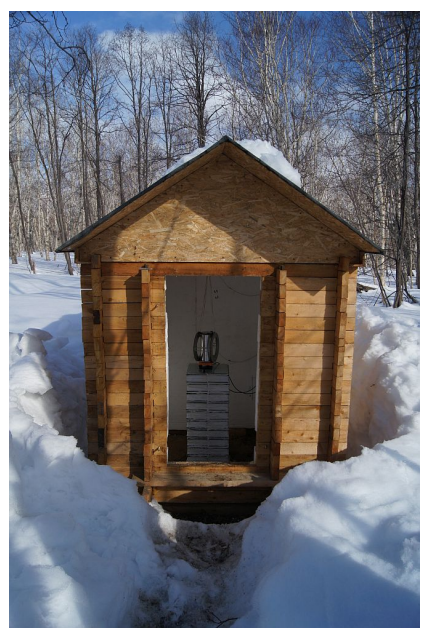

(a)

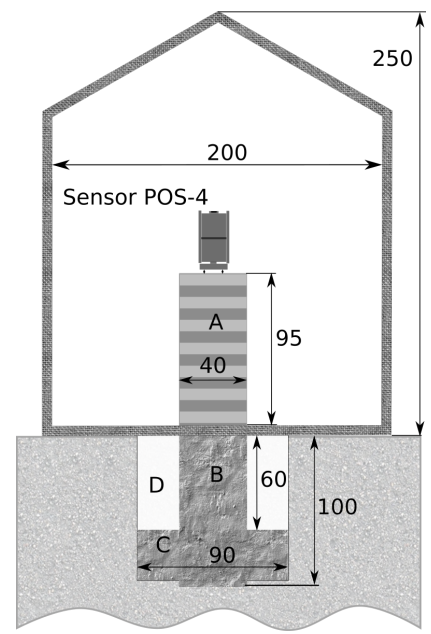

(b)

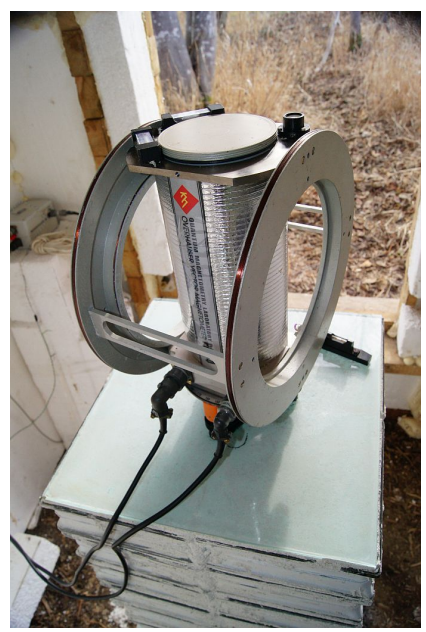

(c)

Figure 3. Location of the magnetometer POS-4 at the Observatory Paratunka: (a) the external appearance of the pavilion; (b) a design of the pavilion and the pillar (dimensions in $\mathrm{cm}$ ); (c) a sensor on the pillar, the level for rough control of the pillar slopes is visible on the right.

is presented for 12.05.2021, when the magnetic field was disturbed (the K-index reached 6). As can be seen, in the differences of the $\mathrm{Z}$ and F values of POS-4 and FGE, slow variations are insignificant. The difference of $\mathrm{Y}$ shows a daily variation up to $2 \mathrm{nT}$, which is due to the influence of temperature on the POS-4 measurements. Also, noticeable deviations of the differences from the constant level are clearly visible in the intervals when the field changed rapidly. This is due to the significant time of measuring the full field vector using POS-4 (10 seconds) and the peculiarities of calculating the components Y and Z. Similar effects are also observed in the records of the dIdD GSM-19FD magnetometer (GEM Systems).

The long-term stability of the magnetometer is controlled by the baselines values of $F_{0}, Z_{0}, Y_{0}$, i.e. by comparing the results of its measurements with the results of absolute observations at the observatory performed manually using the POS-1 scalar magnetometer and the LEMI-203 DIflux magnetometer (LC ISR, Ukraine). Figure 5 shows the average daily baseline values since October 2018. The starting date was chosen from the moment when the pillar stabilized after strengthening, the drift on the old pillar during the freezingdefrosting periods reached several nT per day. The data in figure 5 show that POS- 4 gives fairly stable results in the last three years of continuous measurements. Seasonal variations with a scatter of $0.6 \mathrm{nT}$ are clearly visible only in $F_{0}$, the reason is not established. Similar $F_{0}$ oscillations with a scatter of up to $2 \mathrm{nT}$ are present in the dIdD magnetometer baselines and with a scatter of up to $0.8 \mathrm{nT}$ in the GSM-90 scalar magnetometer baselines, all magnetometers are installed in different pavilions. We can also note the increased dispersion of $Z_{0}$ annually in the winter period, which is also manifested in $Z_{0}$ of dIdD.

\section{The expedition TRANSARCTIC-2019: the works of AARI}

Due to the growing development of the polar regions, geophysical interests here are of great scientific and practical interest. There are only a small number of coastal stations in the auroral zone of Russia, which are not enough to study the geophysical phenomena in this extended territory. There is only one station Spitsbergen in the polar cap area. There is a station on 

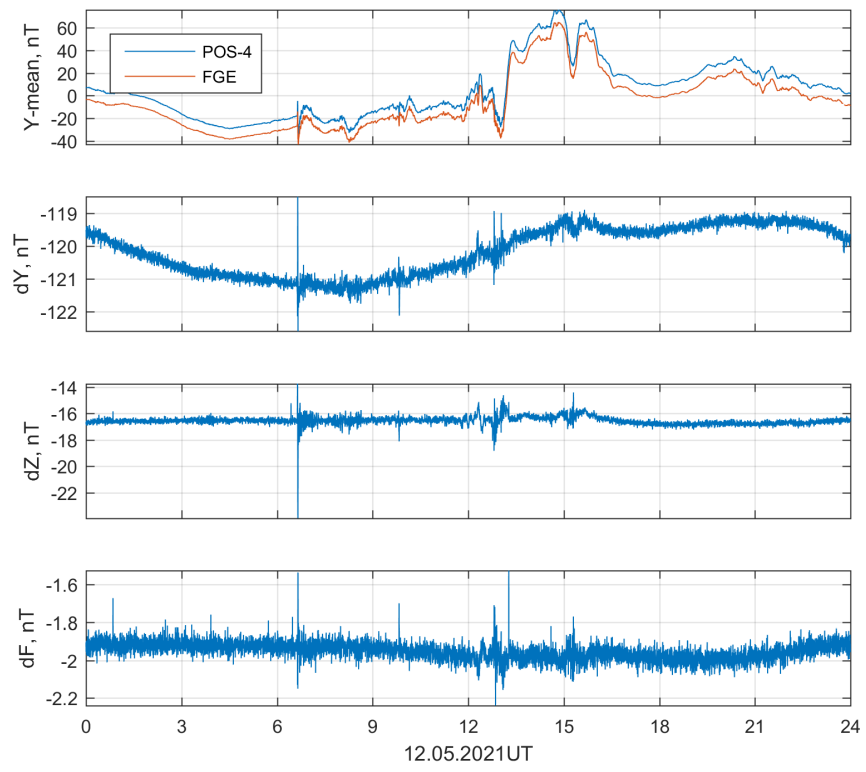

Figure 4. POS-4 and FGE-DTU data for 12.05.2021. The upper panel is values of component $Y$ for POS-4 and FGE (the average values for the day are removed), the lower panels are the differences of POS-FGE of the components Y, Z, F.

Heys Island, which passes into the polar cap zone from the aurora zone only at certain intervals. For the polar cap, where the geophysical processes differ significantly from processes in the auroral zone, such a number of stations is not enough. Significant additional information can be provided by geophysical measurements during the drift through the Arctic Ocean a vessel frozen in ice. The first experience of joint geophysical studies on drifting ice was carried out during of the TRANSARCTIC-2019 expedition. The magnetic field measurements were included in scientific program of expedition along with other geophysical observations.

The magnetometer POS-4 was chosen to measure the magnetic field, since this device is close to the accuracy requirements of the INTERMAGNET standards, and its declared characteristics fully correspond to the conditions that can be created on the ice at a distance from the base vessel. This was confirmed by the fact that the scalar modification of such a device (POS-1) has been used by the AARI at the Cape Baranov polar station for several years without any problems.

It was also taken into account that the magnetometer is a component device, equipped with a tilt meter and allows quickly correct the orientation of the axis of the horizontal component coils during drift and possible reversal of the ice floe. That is, it is possible to measure the total field and the vertical intensity and to calculate the horizontal component even with small changes in the position of the POS-4 solenoid relative to the local vertical. The using of the modern navigation systems in conditions of drifting ice allows to measure the magnetic declination, but this expedition failed to do this. Before the start of the expedition, POS-4 passed a small test on the base AARI "Ladoga" (see figure 6a).

The magnetometer was placed in a non-magnetic heated pavilion with non-freezing design for its emergency evacuation. The pavilion was made in St. Petersburg and then de- 

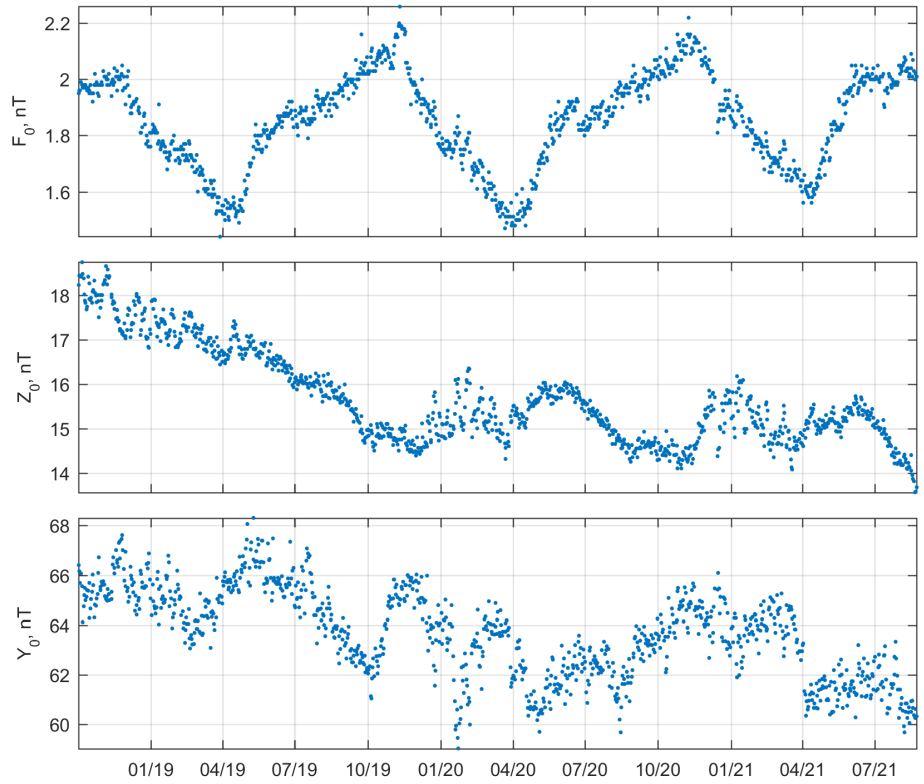

Figure 5. Daily mean baseline values of $F_{0}, Z_{0}, Y_{0}$ of the magnetometer POS-4 since October 2018.

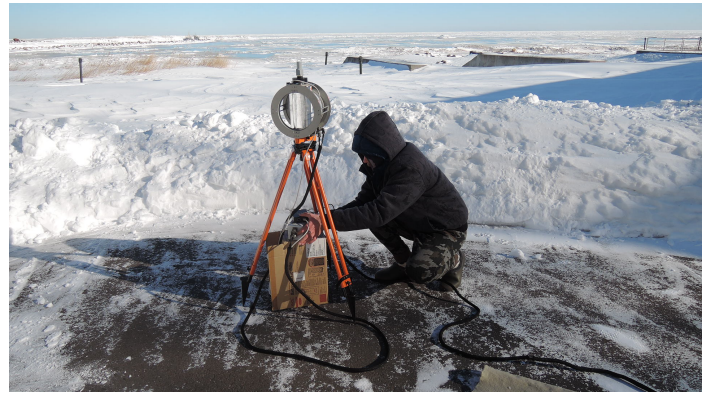

(a)

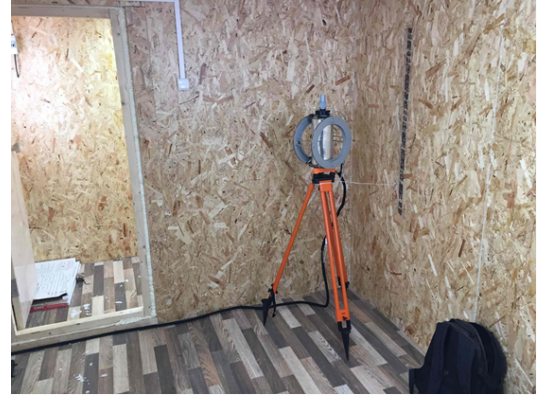

(b)

Figure 6. Magnetometer POS-4 in the TRANSARCTIC-2019 expedition: (a) the testing at the Ladoga field base of AARI; (b) a magnetometer sensor in a non-magnetic pavilion on ice.

livered by ship and helicopter to the installation site on the ice at a distance of about $400 \mathrm{~m}$ from the ship frozen in the ice. The magnetometer was installed in one of the corners of the pavilion on a tripod attached to the elements of the pavilion structure. The device is oriented vertically. The initial orientation of the device to the magnetic north is performed using a compass. The electronics unit and the laptop for data recording were located in the opposite corner of the pavilion (figure $6 \mathrm{~b}$ ).

In continuous operation, the measurements of the total field and vertical intensity of the magnetic field were recorded with rate of the full measurement cycle of 15 seconds. The results of the tilt meter measurements also were recorded. A Wi-Fi connection was established between the pavilion and the ship, which made it possible to constantly monitor the operation 
of the device, reducing the need to visit the pavilion to a minimum. During the entire time of the measurements, there were three failures of the tilt meter, which were probably caused by too large values of angular acceleration caused by the movements of the observer who was in the pavilion. These failures required a restart of the magnetometer control system. Every day, the data was sent to the Polar Geophysical Center of AARI.

Magnetic measurements were carried out in the period from 02 to 27 April 2019. At that time, the station was located at area with geomagnetic latitudes from $68^{\circ}$ to $75^{\circ} \mathrm{N}$, that is, part of the time the station was in the zone of the aurora and for some time was part of the polar cap area. During the drift of the station, the storm magnetic activity was low, but it changed noticeably in the polar cap (figure 7). Figure 7b shows that the values of the PC index, which are a numerical characteristic of the disturbance of the magnetic field in the polar cap, were not very large. Such changes in the magnetic disturbance will allow us to trace the features of the development and flow of magnetic substorms both in the area of the aurora and in the polar cap.
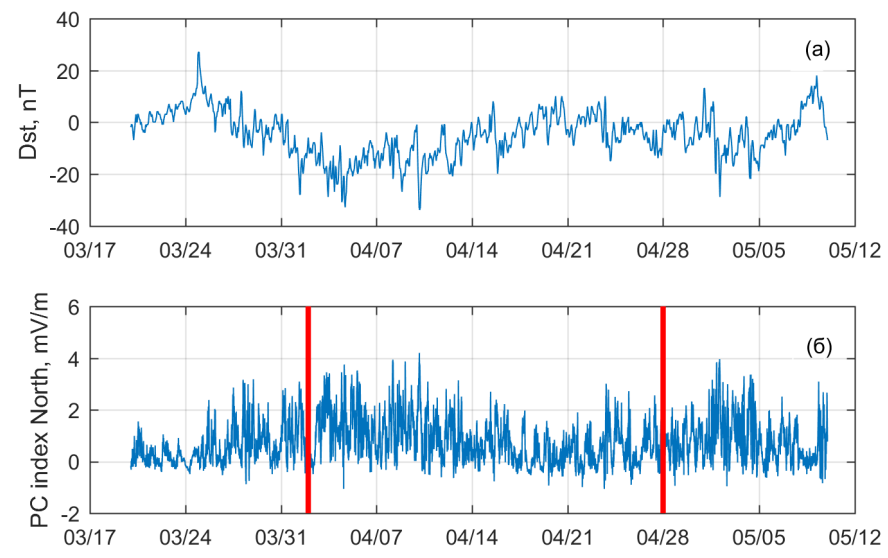

Figure 7. Global magnetic activity during TRANSARCTIC-2019: (a) Dst index (http://wdc.kugi.kyoto-u.ac.jp/dstae/index.html); (b) Polar caps index (PC North, ISGI, http://isgi.unistra.fr). Vertical lines mark the beginning and end of measurements using POS-4.

As an example, in figure 8 the initial records of the variations $\mathrm{dZ}, \mathrm{dH}(\mathrm{F}, \mathrm{Z})$ and $\mathrm{dE}$ (the eastern component, the exact orientation of the $\mathrm{Y}$ axis is not determined) are shown in comparison with the second data obtained at the Hornsund Observatory (HRN, Svalbard Island, Norway, the distance to the expedition is about $800 \mathrm{~km}$ ). As can be seen, there is a consistency of some features in the horizontal components of the field, a less expressive picture for variations of the vertical component. The POS-4 record is quite clean, the most noisy is the $\mathrm{H}$ component, which is calculated from $\mathrm{F}$ and $\mathrm{Z}$.

The calculation of the elements of the Earth's magnetic field using the WMM-2015 model for the 2019.25 epoch at the expedition site and at the HRN observatory (for comparison) are presented in table 1.

One of the problems that arose during the expedition was related to operational processing — daily files that are generated by the POS Manager software have a format that, when read, creates 5 duplicate values for each field element. When standard visualization and analysis tools, such as Excel spreadsheets, are used there are steps on the plots that create discomfort when viewed. An implicit problem in this case is the separation of the measurements of 

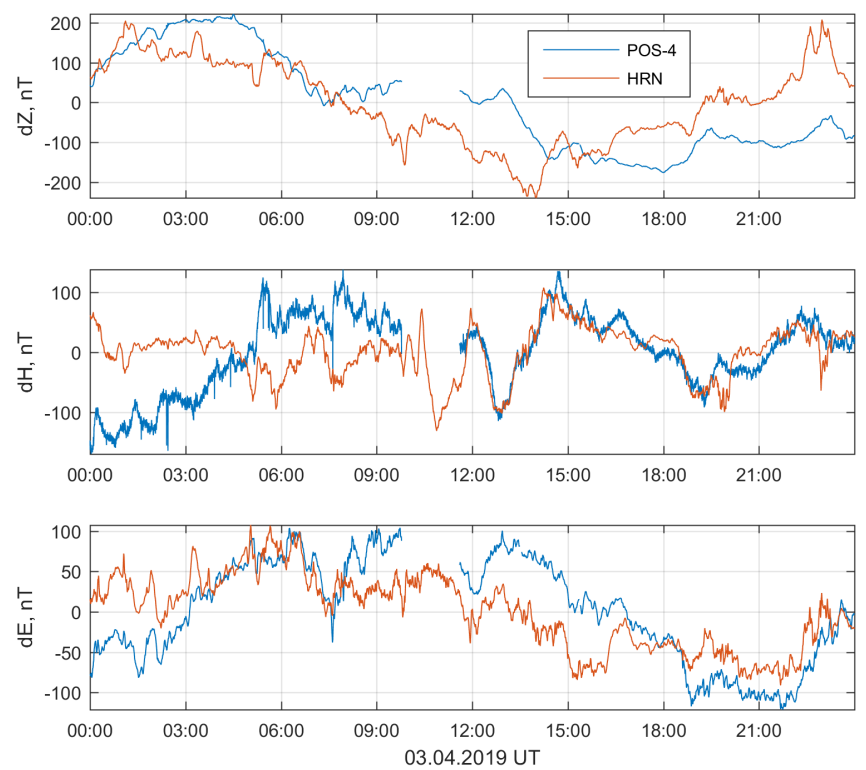

Figure 8. Comparison of the measurement results using POS-4 in the drift ice area and obtained at the HRN observatory for 03.04.2019. The average values are removed.

each of the five total field intensity values by the time in the cycle (for example, when the interval between the measurements is 3 seconds then the full cycle takes 15 seconds). The calculation of field elements (see section 1) assumes that each element is calculated by three total field intensity values that are measured simultaneously. With rapid field variations, this requirement can be significantly violated, up to the occurrence of uncertainties of the form $0 / 0$ or $\sqrt{-1}$.

This may not be important for stationary measurements at the observatories of middle and low latitudes, but it is inconvenient for measurements in the polar regions as well as on a moving (albeit very slow) platform. It should be noted that the problem of simultaneous performance of component measurements by the method of an additional magnetic field was solved when conducting component absolute aeromagnetic surveys. For this purpose, a three-component magnetometric system was created in LO IZMIRAN together with LITMO. It consisted of three quantum magnetometers, the sensors of which were placed in three Braunbeck coil systems, of an optical sensor alignment system, and also included a navigation system, a system for recording and processing. The system was located on a specially equipped AN-12 aircraft. In the case of uniformity of the magnetic field at the site where the measurements were carried out, a fourth magnetometer could be used in the complex to simultaneously measure both the total field and the components while ensuring their mutual influence [12]. In general, there are no significant remarks on the operation of the magnetometer POS-4 in the TRANSARCTIC-2019 expedition. It proved to be a very reliable device, suitable for operation in the climatic conditions of the polar region. 
Table 1. Comparison of Earth's magnetic field elements from observations and from model WMM

\begin{tabular}{ccrrrr}
\hline & data & F & Z & H & Y \\
\hline Transarctic-2019 & WMM & 56157 & 55913 & 5224 & 2595 \\
& POS-4 (03.04.2019) & 56213 & 55975 & 5169 & \\
\hline Hornsund & WMM & 54634 & 54069 & 7839 & 1227 \\
& HRN $(03.04 .2019)$ & 54567 & 53991 & 7905 & 1015 \\
\hline
\end{tabular}

\section{The Geophysical Observatory Arti, IG UB RAS, Arti, Sverdlovsk region}

The Geophysical Observatory Arti of the Institute of Geophysics of the Ural Branch of the Russian Academy of Sciences is located on the outskirts of the urban village Arti, $180 \mathrm{~km}$ from Yekaterinburg.

The close location of the observatory to the Laboratory of Quantum Magnetometry (Ekaterinburg), the presence of a developed infrastructure and various types of magnetometers operating in according to INTERMAGNET standards, and a low background noise level makes it an excellent place to test experimental QMLab developments, calibrate and compare magnetometers, check in real measurements, see figure 9d ([13]).

Since November 2011 the POS-3 No.1 Overhauser ZT magnetometer, a prototype of the POS-4 serial magnetometer, are used at the Observatory Arti. POS-3 allows measuring the vertical and total field intensity. The main tasks for POS-3 were field measurements, including long-term measurements at the Repeat Stations when mapping the Manchazh gravimagnetic anomaly (Ural mountains) on an area of $50 \times 50 \mathrm{~km}$. Between field seasons, POS-3 is installed at the observatory for continuous measurements and various experimental observations. The general view of POS-3 on Repeat Station is shown in figure 9a. From January 2021 the observatory operates POS-3 No.5, modification ZT of the ordinary POS-4 (without horizontal coil system).

Long-term observations on Repeat Station using POS-3 made it possible to estimate the secular variation of the magnetic field on the Manchazh anomaly and to construct the magnetic maps (see figure 10a).

Joint researches of the QMLab developers and Observatory Arti magnetologists of POS4 magnetometers allowed to detect a number of effects, both significant and less obvious in various modifications of Overhauser magnetometers. For example, an important parameter of vector POS measurements is the verticality of the axis of the solenoid in different positions relative to geographical directions and the alignment of this axis with the directions of additional fields generated by the solenoid. Regularly performed studies (figure $9 \mathrm{~b}$ ) produced the error circular diagrams (see an example in figure 10b). These diagrams allowed the developers 


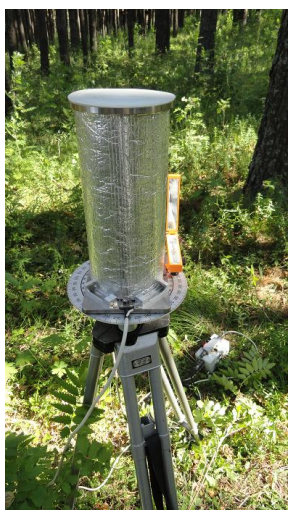

(a)

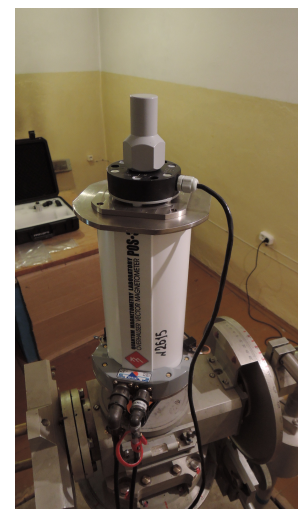

(b)

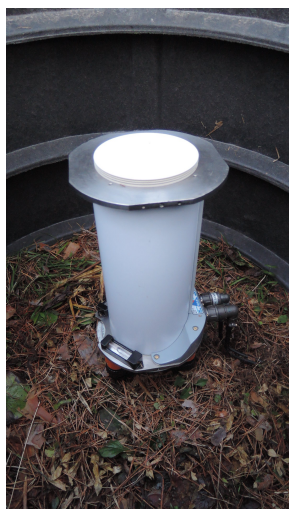

(c)

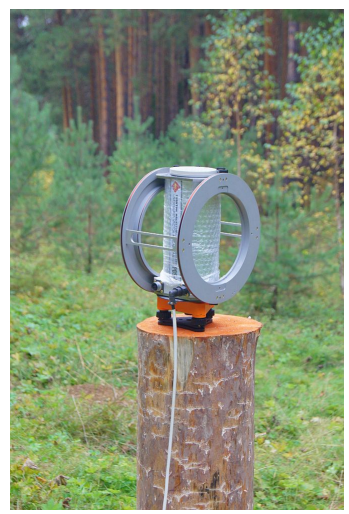

(d)

Figure 9. Vector Overhauser magnetometers POS of QMLab at the Observatory Arti: (a) ZTmagnetometer POS-3 No.1 during work on Repeat Station point; (b) component Overhauser magnetometer POS-3 No.5 with a digital tilt meter on a test stand in a special verification pavilion; (c) POS-3 No.5 outdoors in a protective casing; (d) POS-4 No.3 during the intercomparison of scalar magnetometers at the observatory Arti in 2017 [13].

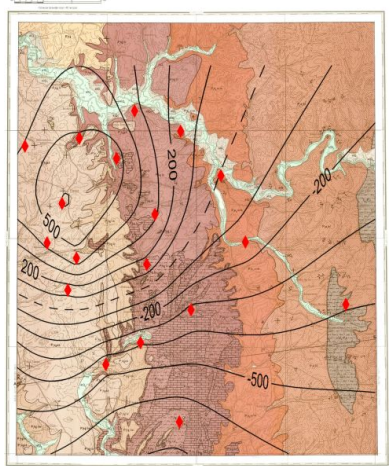

(a)
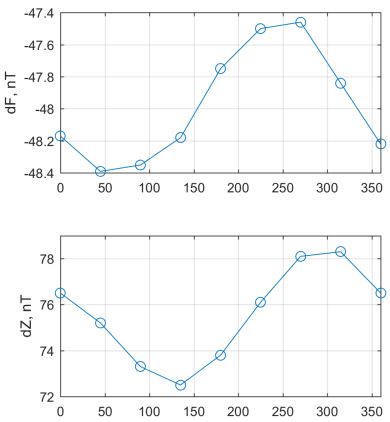

(b)
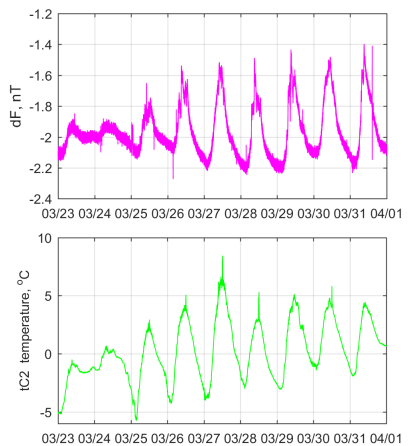

(c)

Figure 10. Some results of researches using POS-3 and POS-4 magnetometers: (a) the anomalous secular variation of the vertical component of Earth's magnetic field for the epoch 2019.5. The isodynamic lines are drawn through $100 \mathrm{nT}$; (b) a circular diagram for the total field and vertical intensity of POS-3 No.5; (c) presumably, the thermal effect in the titanium solenoid appeared as the difference between the F of POS-3 No.5 and POS-1 against the outdoor temperature.

to improve above mentioned POS-4 parameters by almost an order of magnitude. Currently, the systematic error when rotating the device in the azimuthal plane does not exceed $1 \mathrm{nT}$ for $\mathrm{F}$ and $6 \mathrm{nT}$ for $\mathrm{Z}$.

As the magnetometer POS-4 has shown at the Observatory Paratunka, the results of measurements significantly depend on the stability of the base on which the magnetometer sensor is installed [7]. This dependence, for example, in the Canadian dIdD GSM-19FD magnetometer, is partially solved by placing the sensor and the coil system at the gimbal. In modern POS-4 models, a different approach is implemented - the slopes of the solenoid are 
recorded using a digital tilt meter (see figure 9b) and appropriate corrections are applied to the magnetic data.

It is noted that the POS-1 scalar sensor in a titanium solenoid is sensitive to temperature gradients. This is especially evident when working in the field with outdoor place of sensor. The sensitivity of POS-4 to the outdoor temperature in spring, when working on frozen ground, was studied at the observatory Arti. The device is mounted on an aluminum rod, sunk a meter into the frozen ground. The magnetometer is protected from direct sunlight by a plastic casing (see figure 9c). The results are shown in figure 10c.

\section{Magnetometers POS-4 at the Observatory Moscow, IZMIRAN, Troitsk}

IZMIRAN acquired two POS-4 Overhause vector magnetometers at the end of 2020, including POS-4 (No.5) for the Observatory in Kaliningrad, and the second (No.6) for the magnetic station in the village Vaimusha, Pinezhsky district, Arkhangelsk region. Before being sent to the station Vaimusha the magnetometer No.6 was installed at the IZMIRAN Observatory Moscow in Troitsk in the absolute pavilion (figure 11a) and tested for several months in a continuous measurement.

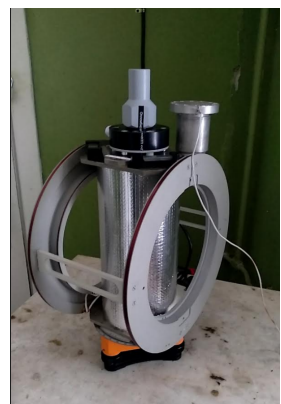

(a)

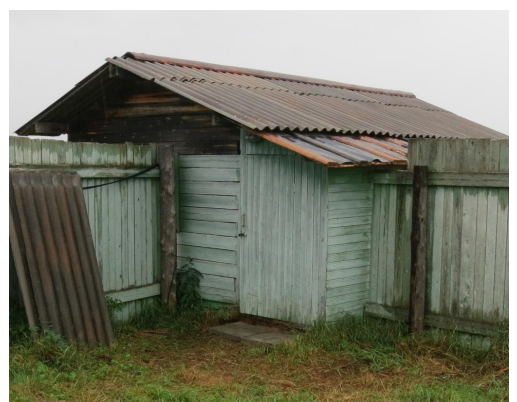

(b)

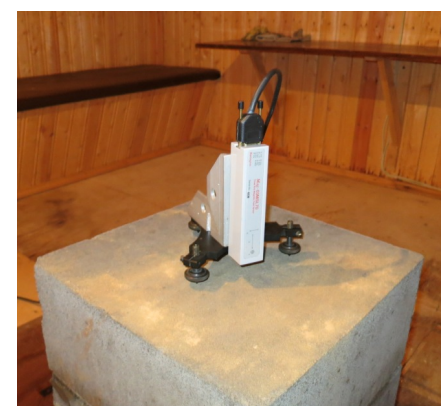

(c)

Figure 11. POS-4 magnetometer (IZMIRAN): (a) a testing of POS-4 in the absolute pavilion of the Observatory Moscow; (b) a pavilion for installing of POS-4 at the IZMIRAN station in the village Vaimusha; (c) a pillar inside the pavilion with a fluxgate magnetometer.

As an example, the figure 12 shows the comparison of the raw second values of the variations of the Y- and Z-components obtained using POS-4 and the data of the LEMI018 fluxgate magnetometer installed at the observatory Mikhnevo at distance of $80 \mathrm{~km}$ from IZMIRAN. The observatory Mikhnevo was chosen for comparison due to a more favorable noise environment than in Troitsk. The middle panels show the differences between the data of two magnetometers, the lower panel shows the parameter QMC (for measurements with two opposite magnetizing fields). As can be seen in figure 12 the results of POS-4 are close to the results of the observational magnetometer, but they are more noisy during the day (primarily in the vertical component), with the exception of a small interval of 1-2 hours in the night local time. It can also be noted that the parameter QMC increases approximately every 8 hours, which indicates a regular source of noise near the measurement place.

The estimation of long-term stability was performed by comparing the hourly mean values of POS-4 and the hourly mean data obtained by the standard quartz variometer of the Observatory Moscow. Figure 13a shows the hourly values of the Y- and Z-components from June 1 to June 20, 2021, figure 13b shows the differences of these curves. Figure 13b also 

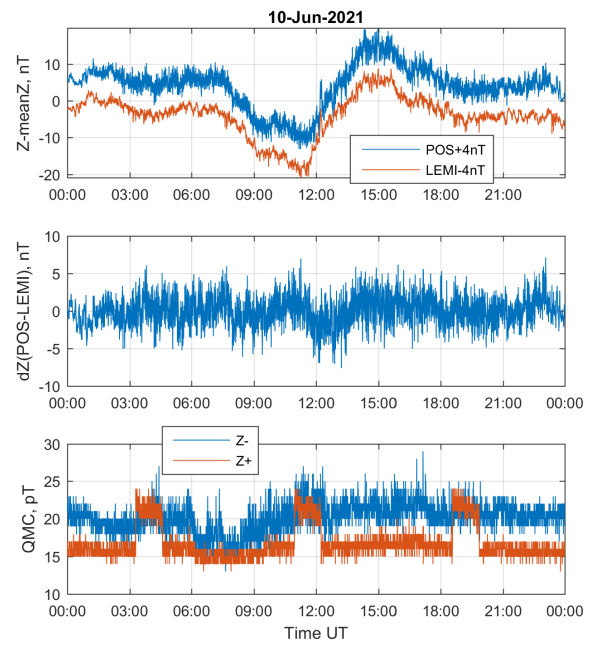

(a)
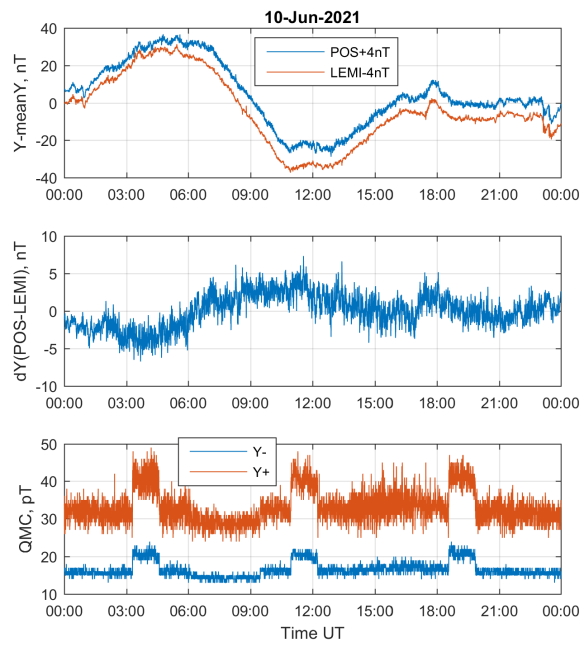

(b)

Figure 12. Example of measurements by POS-4 for 10.06.2021, components $Z$ (a) and Y (b), a full measurement cycle of 15 seconds. For comparison, the raw 1-second data obtained at the Observatory Mikhnevo using the variometer LEMI-018 is presented.

shows variations in the inclination of the solenoid in the "north-south" and "east-west" directions. As can be seen from the curves in figure 13b, there are no slow drifts in the POS-4 data, however, on some days there is a scatter in the differences, probably associated with increased geomagnetic activity. There is also no correlation between the tilts of the POS-4 solenoid and the magnetic data.

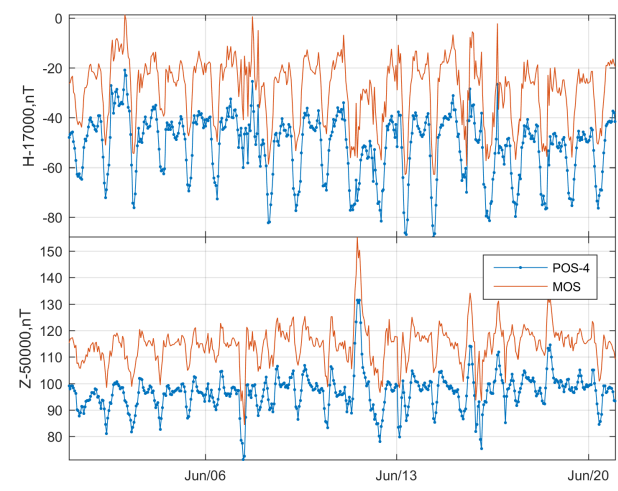

(a)

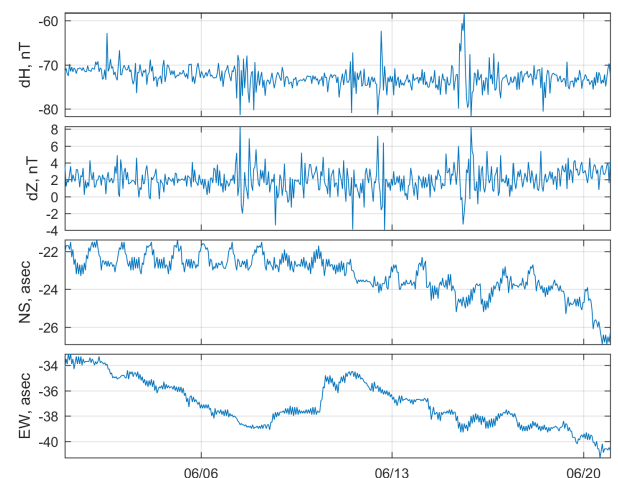

(b)

Figure 13. Hourly mean data recorded by the POS-4 and by the Observatory Moscow variometer (a) and its differences and tilts of the POS-4 solenoid (b).

In August 2021, the staff of the Moscow Magnetic Observatory prepared a place for the installation of POS-4 at the IZMIRAN station in the village Vaymusha. As a result of these works, a pavilion was prepared, a pillar was installed inside. Figures $11 \mathrm{~b}, \mathrm{c}$ shows the pavilion 
itself and a pillar with a three-component fluxgate magnetometer, which was used to check the suitability of the selected place for magnetic measurements. The installation of POS-4 on a permanent place is planned for the next visit. To improve the conditions for long-term autonomous measurements, it is planned to install a heated polypropylene screen along the perimeter of the pillar with POS-4.

IZMIRAN plans to use magnetometers POS-4 for continuous and largely autonomous measurements. Long-term testing at the Moscow observatory showed that the magnetometer works quite stably, but several times there were failures (measurement stops) that required the some work of an observatory employee to manually restart the device. The cause of the failures has not been established. This moment becomes critically important for long-term autonomous measurements. There were also some problems with the software when writing files.

\section{Magnetometers POS-4 at the Observatory White Sea, GC RAS}

The White Sea Magnetic Observatory (WSE) was organized by the Geophysical Center of RAS on the base of the White Sea Biological Station of Moscow State University: a nonmagnetic pavilion with a glass block pillar was built on a specially selected site during June 2018, and a magnetometer POS-4 was installed and continuous measurements began in August 2018. POS-4 works almost completely independently, data is transmitted online to the GC RAS (Moscow) and is available on the website http://geomag.gcras.ru/dataprodplot.html. Photos of the pavilion and the sensor of POS-4 are shown in figure 14. The pavilion for POS-4 is not heated, i.e. the magnetometer is located in quite extreme conditions. Maintenance is performed sporadically, by employees of the GC RAS.

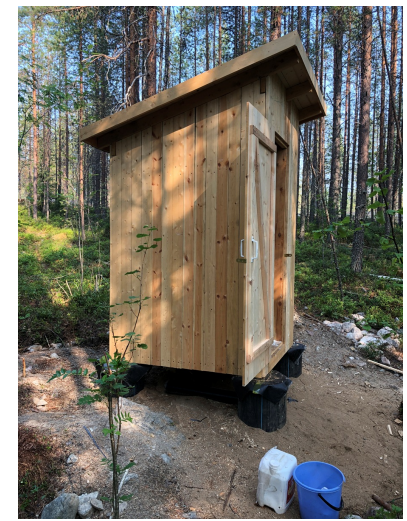

(a)

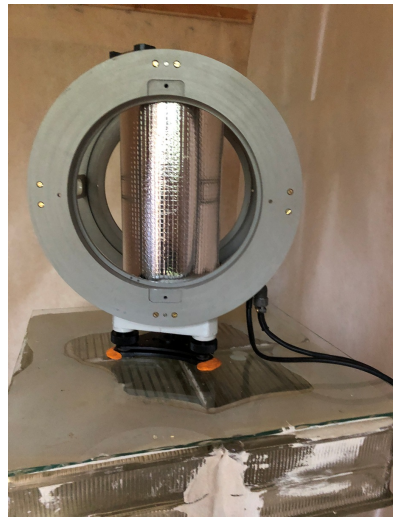

(b)

Figure 14. Magnetometer POS-4 at the White Sea Magnetic Observatory (WSE) of the GC RAS: (a) the view of the non-magnetic pavilion (under construction), (b) the POS-4 sensor on a pillar (http://geomag.gcras.ru/obs-WSE.html).

As an example, figure 15 presents the 1-second data (the full measurement cycle is 15 seconds) obtained by POS-4 at the White Sea Observatory, compared with the data of the nearest auroral observatories Abisko (ABK) and Lycksele (LYC), Sweden. As can be seen from the figure, the variations of the X-component obtained using POS-4 are structurally close to the variations at the ABK and LYC observatories, it is hardly possible to expect a coincidence of details in the magnetic field variations at spatially separated points in the 
auroral area. However, in the highlighted three-hour fragment shown in the lower panel of 15 , it is possible to notice the synchronous manifestation of some features.
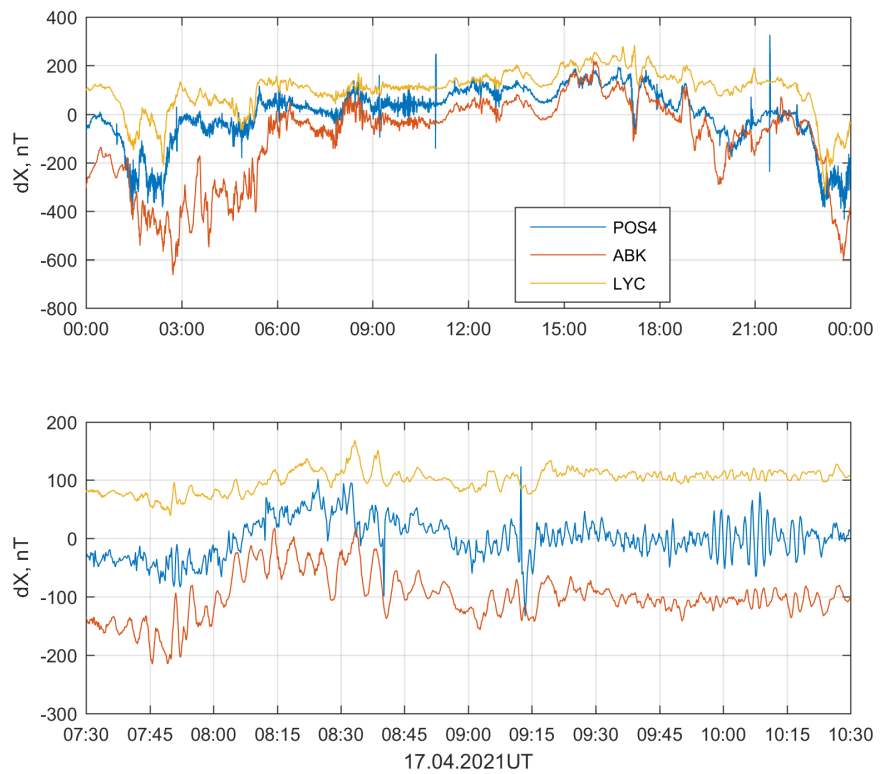

Figure 15. Comparison of the X-component variations obtained using POS-4 at the WSE observatory and at the INTERMAGNET ABK and LYC observatories (1-second values). The upper panel shows the variations for the day of 17.04.2021, the lower panel is a part of the data from 07:30 to 10:30UT, showing some details of the variations.

The three-year experience of using POS-4 at the White Sea Observatory has shown that the main problems are episodic stops of measurements (the reason is not established, but probably occurs with strong noise) and a large number of significant amplitude spikes. Some of this noise can be seen in figure 15, their amplitude reaches hundreds of nT. Presumably, the source of this noise is the power plants used at the White Sea Biological Station.

\section{Discussion and conclusion}

Vector Overhauser magnetometer POS-4, developed at the Quantum Magnetometry Laboratory of the Ural Federal University (Yekaterinburg), is the result of combining long-known principles and methods of measuring elements of the magnetic field vector and modern technologies, element base and microprocessor systems. Many years of experience in using POS4 at observatories and on expeditions allowed us to identify the problem areas of the magnetometer. This made it possible for developers to make methodological, technical, design and software changes to the device that improve its characteristics.

The undoubted advantages of the POS-4 magnetometer include:

1) structural reliability, compactness, absence of moving parts and elements made of brittle materials - this makes the magnetometer suitable for field work, in which the probability of mechanical damage to the equipment significantly increases; 
2) easy to install, configure, run measurements and monitor results - this becomes very important when POS-4 are used in the absence of personnel with a sufficient level of qualification;

3) POS-4 allows us to obtain the elements of the magnetic field $F$ and $\mathrm{Z}$ (and $\mathrm{H}$ ) in the absolute sense, but with the frequency of variational measurements. In fact, there is a device that performs two functions that are mandatory for INTERMAGNET observatories. A methodological question remains open with the absoluteness of the horizontal component measured using a coil system;

The main problems for POS-4, which were discovered during POS-4 operation, were:

1) a significant dependence of the measurement results on the stability of the base (the slopes of the solenoid and the turns of the coil system). The problem was manifested in long-term trends in observational conditions, for example, at the Paratunka observatory during seasonal freezing and thawing of the soil under the pillar, or in field conditions when measurements were performed from a tripod. As a solution, it was decided to use a highprecision tilt meter, the non-magnetic sensor of which could be fixed on the upper part of the magnetometer solenoid. A similar method of accounting for tilts is used, for example, in fluxgate magnetometers MAGDAS [14]. Currently, a method for compensating errors due to tilts has been developed and it is being implemented in the POS-4 software. Unfortunately, the control of rotations of the POS-4 sensor around the vertical, i.e. effects in horizontal components, remains open;

2) a methodological problem related to the choice of the orientation of the coil system fixed on the solenoid and the orientation of the scalar Overhauser sensor POS inside the solenoid. The orientation of the axis of this coil system determines which component in the horizontal plane will be determined. The orientation of the primary sensor with significant magnetizing fields in the vertical plane can increase the POS-4 intrinsic noise. Currently, the priority is to measure the eastern component and the orientation of the axis of the sensor POS perpendicular to the magnetic meridian. A similar problem, for example, is absent in the dIdD GSM-19FD, since the orientation relative to the field vector of pairs of declination and inclination coils is performed by aligning the readings in the measured channels $(\mathrm{D}+$, D-) and (I+, I-);

3) there is a problem with the solenoid stand used, since its size does not provide sufficient stability on the pillar and sufficient sensitivity of the support screws when levelling. In addition, there is no possibility of precise rotation of the solenoid around the vertical axis (when studying a circular diagram or the orientation of the horizontal axis of the coil system), as is done, for example, with dIdD. Unfortunately, a possible solution by increasing the base of the stand and complicating its mechanical components may be acceptable only for the conditions of observatories and is not suitable for the field version;

4) the format used for writing data to files by the standard POS Manager program contains all the information that is obtained during measurements, including the measurement of each of the five modules is marked with its own time stamp. However, a certain problem arises for users who do not have the opportunity to develop their own software that would provide convenient work with data and minimize some methodological errors. Currently, as the experience of working with POS-4 in IZMIRAN shows, some changes have been made to the POS-4 file format, but it is not yet possible to assess how successful they are.

The tasks of automation of absolute magnetic measurements, including the creation of hybrid systems that combine the functions of absolute and variation magnetometers, remain relevant. In our opinion, the important aspects of these developments are the control of the spatial orientation of the measuring axes, noise immunity and ease of operation. The experience of using vector Overhauser magnetometers POS-4 and its ZT prototype POS-3 for several years shows that trends in modifications of these devices reflect the requests of mag- 
netologists in the tasks of observing the Earth's magnetic field. The balance of achievements and shortcomings of the magnetometer POS-4 shown in this work gives reason to hope that in the future we can expect the appearance of a device suitable for the effective solution of a complex of scientific and applied problems, including observations under the Arctic and in tectonically active regions conditions.

\section{Acknowledgments}

The authors thank the employees of observatories and institutes who supported the operation of POS-3 and POS-4 magnetometers. The results presented in this paper rely on data collected at magnetic observatories. We thank the national institutes that support them and INTERMAGNET for promoting high standards of magnetic observatory practice (www.intermagnet.org). Work of S.Y. Khomutov was supported by budget project AAAAA21-121011290003-0, work of P.B. Borodin, A.S. Bebnev and T.A. Cherepanova was supported by budget project AAAA-A19-119021290090-4, work of Y.G. Turbin and P.E. Baryshev was carried out as a part of the AARI planned scientific topic under the project 5.1.4 of the Targeted Scientific and Technical Program (TSTP) of Roshydromet.

\section{References}

[1] INTERMAGNET Technical Reference Manual. Version 5.0.0. St-Louis B. (Ed.) (INTERMAGNET Operations Committee and Executive Council, 2020) 156

[2] J.L. Rasson, A. Gonsette, Data Science Journal 10 IAGA169-IAGA173 (2011)

[3] S. Marsal, J.J. Curto, J.M. Torta, A. Gonsette, V. Favà, J. Rasson, M. Ibañez, Ò. Cid, Geosci. Instrum. Method. Data Syst. 6 269-277 (2017)

[4] L. Hegymegi, J. Szöllősy, C. Hegymegi, Á. Domján, Geosci. Instrum. Method. Data Syst. 6 279-284 (2017)

[5] V.I. Myukhkyurya, Geophys. Equip. (in Russian) 31 NP (1967)

[6] dIdD Insrtuction Manual v7.0, Release 7.5 (GEM Systems, ON Canada, 2010) 50

[7] S. Khomutov, V. Sapunov, A. Denisov, D. Savelyev, I. Babakhanov, E3S Web of Conferences 11,00007 1-5 (2016)

[8] A.Yu. Denisov, V.A. Sapunov, O.V. Dikusar, Geomagnetism and Aeronomy 39/6 737$742(1999)$

[9] A.Y. Denisov, V.A. Sapunov, B. Rubinstein, Meas. Sci. Technol. 25/055103 1-6 (2014)

[10] V.A. Sapunov, A.Y. Denisov, D.V. Saveliev, A.A. Soloviev, S.Y. Khomutov, P.B. Borodin, E.D. Narkhov, A.V. Sergeev, A.N. Shirokov, Magnetic Resonance in Solids. Electronic Journal 18/2 1-9 (2016)

[11] S.Y. Khomutov, E3S Web of Conferences 20,02002 1-18 (2017)

[12] Y.G. Turbin, V.I. Pochtarev, V.I.Yuschenko, N.V. Alekseev, E.A. Bugrov, N.G. Ptitsina, A.Y. Rotshtein, M.A. Sergeev, A.D. Cherednichok, Geomagnetism and aeronomy (in Russian) 17,2 326-331 (1977)

[13] O.A. Kusonsky, S.Y. Khomutov, P.B. Borodin, V.A. Sapunov, D.V. Savel'ev, V.D. Savel'ev, E.D. Narkhov, L.A. Murav'ev, A.Y. Gvozdarev, I.A. Nuzhdaev, A.V. Ovcharenko, A.S. Bebnev, Conrad Observatory Journal 523 (2019)

[14] MAGDAS-A Installation Manual, SERC, Kyushu Univ., Editor George N. Maeda, 35 (2005) 